\title{
Isolation of the Histone Lysine Methyltransferase Gene PhSDG8 and Characterization of Function in Shoot Branching
}

\author{
Lili Dong, Tongrui Liu, Di Gao, Jing Li, and Jie Qian \\ Department of Ornamental Horticulture, Anhui Agricultural University, Hefei, 230061, People's \\ Republic of China
}

\begin{abstract}
AdDitional INDEX wORDs. expression analysis, overexpression, subcellular localization, UGT74E2, VIGS
Abstract. Petunia (Petunia $\times$ hybrida) is an important ornamental plant, and its branch development has become a hot research topic. In this study, PhSDG8, an ortholog of SET domain group $8(S D G 8)$, was cloned from the petunia cultivar Mitchell Diploid. It had an open reading frame (ORF) of 5070 bp and encoded 1689 amino acids, with Suppressor variegation 3-9, Enhancer of zeste, Trithorax (SET), Zinc finger-cysteine and tryptophan conserved (ZfCW), associated with SET (AWS) and Post SET domains. The predicted amino acid sequence of PhSDG8 was most closely related to Nicotiana sylvestris ASHH2 (NsASHH2). Expression analysis revealed that PhSDG8 expressed highest in the stems and lowest in the axil. Subcellular localization analysis showed that PhSDG8 was localized in the nucleus. Overexpression of PhSDG8 reduced the branch number of Arabidopsis thaliana sdg8-2. The silencing of $P h S D G 8$ resulted in an increase in the number of branches of petunia and significant upregulation of $P h U G T 74 E 2$. These results suggested that $P h S D G 8$ may be a candidate gene for regulating the branching of petunia.
\end{abstract}

Petunia (Petunia $\times$ hybrida) is one of the most important ornamental plants. In its cultivation, artificial topping is required to promote branch development to achieve the ornamental effects, but this task greatly increases the production cost. Therefore, breeding new cultivars with different branching characteristics through genetic engineering is an important way to solve the efficient production of petunia. At the same time, petunia has the characteristics of easy regeneration and short life cycle, and its genome has been successfully sequenced (Bombarely et al., 2016). Therefore, petunia has become a model plant for branch development and other research.

In recent years, great progress has been made in the branch development of petunia, such as identification of the strigolactone synthesis gene decreased apical dominance 1 (DAD1), DAD3, P. hybrida more axillary buds 1 (PhMAX1), signal transduction gene PhMAX2,DAD2, and transporter gene pleiotropic drug resistance (PDR1) (Drummond et al., 2009, 2011; Hamiaux et al., 2012; Kretzschmar et al., 2012; Snowden et al., 2005). Other studies mainly focus on the start time of the axillary meristem and environmental factors affecting petunia branching (Dal Santo et al., 2011; Drummond et al., 2015; Revel et al., 2015; Zenoni et al., 2011). These studies lay a good foundation for revealing the mechanism of petunia branch development.

However, the regulatory network of branching is extremely complicated. The formation of branches depends on the initiation of axillary meristems and the activity of axillary buds

Received for publication 21 Apr. 2020. Accepted for publication 12 June 2020. Published online 17 July 2020.

This work was supported by The Natural Science Foundation of Anhui Province (1808085MC85), Excellent Young Talents Support Plan of Anhui Province College (gxyq2019010), and the National Natural Science Foundation of China (31902042).

L.D. is the corresponding author. E-mail: dongli0608@163.com.

This is an open access article distributed under the CC BY-NC-ND license (https://creativecommons.org/licenses/by-nc-nd/4.0/).
(Shimizu-Sato et al., 2009). The initiation of axillary meristems is regulated by many genes (Dun et al., 2012). Branchedl (BRC1), known as the class II Teosinte Branched1/Cycloidea/ Pcf (TCP) family of transcription factors, has been shown to be an integrator of multiple internal and external signals to suppress branching (Shen et al., 2019). Regulations of axillary meristems 1 ( $R A X 1), R A X 2, R A X 3$ redundantly regulate axillary bud formation at different stages of shoot development in a daylength-dependent manner (Müller et al., 2006). Lateral suppressor $(L A S)$ is mainly expressed in a band-shaped domain at the adaxial side of young leaf primordia, and loss of $L A S$ function causes strong defects in axillary meristem (AM) formation (Fambrini et al., 2017). Regulation of axillary of meristem formation $(R O X)$ mRNA accumulates at the adaxial boundary of leaf and flower primordial, and it acts in concert with $R A X 1$ and $L A S$ to modulate AM formation (Yang et al., 2012). WRKY71 affects AM initiation and bud activity by positively regulating the transcription of $R A X 1, R A X 2$, and $R A X 3$ (Guo et al., 2015).

In recent years, it has been found that epigenetics is also an important factor regulating shoot branching. The histone lysine methyltransferase SDG8, as the homologous protein of the histone H3K36 methyltransferase SET2 in fungi (Sui et al., 2013), is also called Absent, Small, or Homeotic Disc 1 (ASH1) homolog 2s (ASHH2). SDG8 was first identified as early flowering in short days (EFS) in Arabidopsis (Arabidopsis thaliana), the mutation of which leads to a phenotype of early flowering (Kim et al., 2005). Later, it was found that SDG8 was able to regulate shoot branching (Cazzonelli et al., 2009; Dong et al., 2008; Liu et al., 2016; Zhao et al., 2005). The current study suggests that SDG8 reduces the trimethyl level of histone 3 lysine $4(\mathrm{H} 3 \mathrm{~K} 4)$ near the transcriptional start position of carotenoid isomerase (CRTISO) and increases the level of dimethylation, which lead to an increase of CRTISO mRNA levels, thereby regulating the content of carotenoids and the synthesis of strigolactone (Cazzonelli et al., 2009). On the other hand, $S D G 8$ can affect shoot branching by regulating the 
Table 1. Primer names and sequences used for polymerase chain reaction amplification.

\begin{tabular}{|c|c|}
\hline Primer name & Primer sequences $\left(5^{\prime}-3^{\prime}\right)$ \\
\hline$B R C 1-R T-F$ & ACTTAAAGAAGGTACAAACTGG \\
\hline$B R C 1-R T-R$ & AAAATAACTGGACTGAAGGGGT \\
\hline$R A X 1-R T-F$ & TGACAAGAACAAAGTGAAAAG \\
\hline$R A X 2-R T-F$ & AAAGAAAATGTAAAAAGGGGT \\
\hline$R A X 2-R T-R$ & GAGATGATAGGTCAAGAGCCA \\
\hline$R A X 3-R T-F$ & AGATGTGGAAAGAGTTGTAGG \\
\hline$L A S-R T-F$ & GTTATCСТCСТCСТАСТСТTCG \\
\hline$L A S-R T-R$ & CACACTTGTTCCACTGTCATTC \\
\hline$R O X-R T-F$ & АTTCTTCTTGTTTATCTTCTTC \\
\hline$R O X-R T-R$ & CACTTATTCTATGCCTTCTTTC \\
\hline WRKY71-RT-F & TTTCTACTATCACCAGCCATT \\
\hline WRKY71-RT-R & ТTСТТСТСТССТТТСТTТТTТ \\
\hline Beta-Actin-F & GTTGGACTCTGGTGATGGTGTG \\
\hline Beta-Actin-R & CCGTTCAGCAGTGGTGGTG \\
\hline Tublin-F & GAGCCTTACAACGCTACTCTGTCT \\
\hline Tublin-R & ACACCAGACATAGTAGCAGAAATC \\
\hline$S D G 8-v i g s-F$ & CGGAATTCCTCTATTCCTACAGGTCCCATATC \\
\hline$S D G 8$-vigs- $R$ & CGGGATCCTTGGGTATTGCTCATACTATCTGGA \\
\hline$S D G 8-F-S L$ & GAGAACACGGGGGACTCTAGAATGGTGCAAGCGGTTGAACT \\
\hline$S D G 8-R-S L$ & GCCCTTGCTCACCATGGATCCCATTCTTGGGTTTTGCGCA \\
\hline$P h S D G 8-R-O E$ & GCCCTTGCTCACCATGGATCCCTACATTCTTGGGTTTTGCGCA \\
\hline
\end{tabular}

expression of SPS/BUS (supershoot/bushy) and UDP-glycosyltransferase 74E2 (UGT74E2) (Dong et al., 2008). As we know, UGT74E2 is an UDP-glucosyltransferase that affects auxin homeostasis (Tognetti et al., 2010). Therefore, it is speculated that SDG8 may regulate the branch development mainly by affecting the dynamic balance of strigolactone and auxin.

In this study, we cloned the petunia homolog gene of $S D G 8$, carried out bioinformatics analysis on its sequence, and detected the expression level of PhSDG8 in different tissues using quantitative real-time PCR (qRT-PCR). Also its subcellular localization and function were analyzed. This study lays a foundation for further research on the mechanism of regulation of branch development in petunia.

\section{Materials and Methods}

Plant materials and Growth conditions. The petunia cultivar Mitchell Diploid was used in this study. Petunia seedlings, the Arabidopsis Columbia wild type (WT), sdg8-2 (Zhao et al., 2005), transgenic plants were all planted in the pots and placed in tissue culture rooms with light-emitting diode lamps (Green Power TLED V5; Philip, Shanghai, China), with the temperature of $23 \pm 2{ }^{\circ} \mathrm{C}$, the illumination of $100 \mu \mathrm{mol} \cdot \mathrm{m}^{-2} \cdot \mathrm{s}^{-1}$, and the photoperiod of $16 / 8 \mathrm{~h}$ (light/ dark).

IsOlation OF THE PHSDG8 GENE. Total RNA was extracted from stems of petunia plants using EZ-10 Total RNA MiniPreps Kit (Sangon Biotech, Shanghai, China). DNA removal and First-strand cDNA synthesis was performed using EasyScript One-Step gDNA Removal and cDNA Synthesis Super-
Mix (Transgene, Beijing, China). Primers were designed according to the petunia genome sequence (Bombarely et al., 2016) (Table 1). The products amplified by using KOD-PlusNeo DNA Polymerase (Toyobo, Shanghai, China) were cloned into pEASY ${ }^{\circledR}$-Blunt Simple Cloning Vector (Transgene) to determine the sequence of PhSDG8.

BioinformatiC ANALYSIs. Based on the published Arabidopsis ASH1s, other ASH1 proteins were obtained from the National Center for Biotechnology Information (NCBI) database by blasting with known amino acid sequences of ASH1s. Multiple sequence alignment was calculated with DNAMAN software (version 5.2.2; Lynnon Biosoft, Quebec, QC, Canada). Phylogenetic analysis was conducted using the MEGA 5.05 program (Tamura et al., 2011) by the neighbor-joining method. A ProParam online tool in ExPASy (Gasteiger et al., 2005) was used to analyze the basic physical and chemical properties of the protein sequences.

EXPRESSION ANALYSIS. For the tissue expression experiments, roots, stems, leaves, axil, and apex of 30-day-old petunia seedlings were collected. We performed qRT-PCR and analysis as described by Dong et al. (2013). SDG8-RT-F/SDG8-RT-R was used to detect the expression level of PhSDG8. $\beta$-actin was used as a normalization control with primers Beta-Actin-F/ Beta-Actin-R. For analysis of gene expression of plants inoculated with pTRV1/pTRV2-0 or pTRV1/pTRV2-PhSDG8, after $60 \mathrm{~d}$ of infiltration, axillaries were taken to extract RNA for reverse transcription, and the expression levels of $P h S D G 8$, PhBRC1, PhRAX1, PhRAX2, PhRAX3, PhLAS, PhROX, $P h W R K Y 71$, and PhUGT74E2 were detected (Table 1).

SubCellular localization analysis. To construct the PhSDG8-GFP fusion protein, $P h S D G 8$ cDNA, not containing 


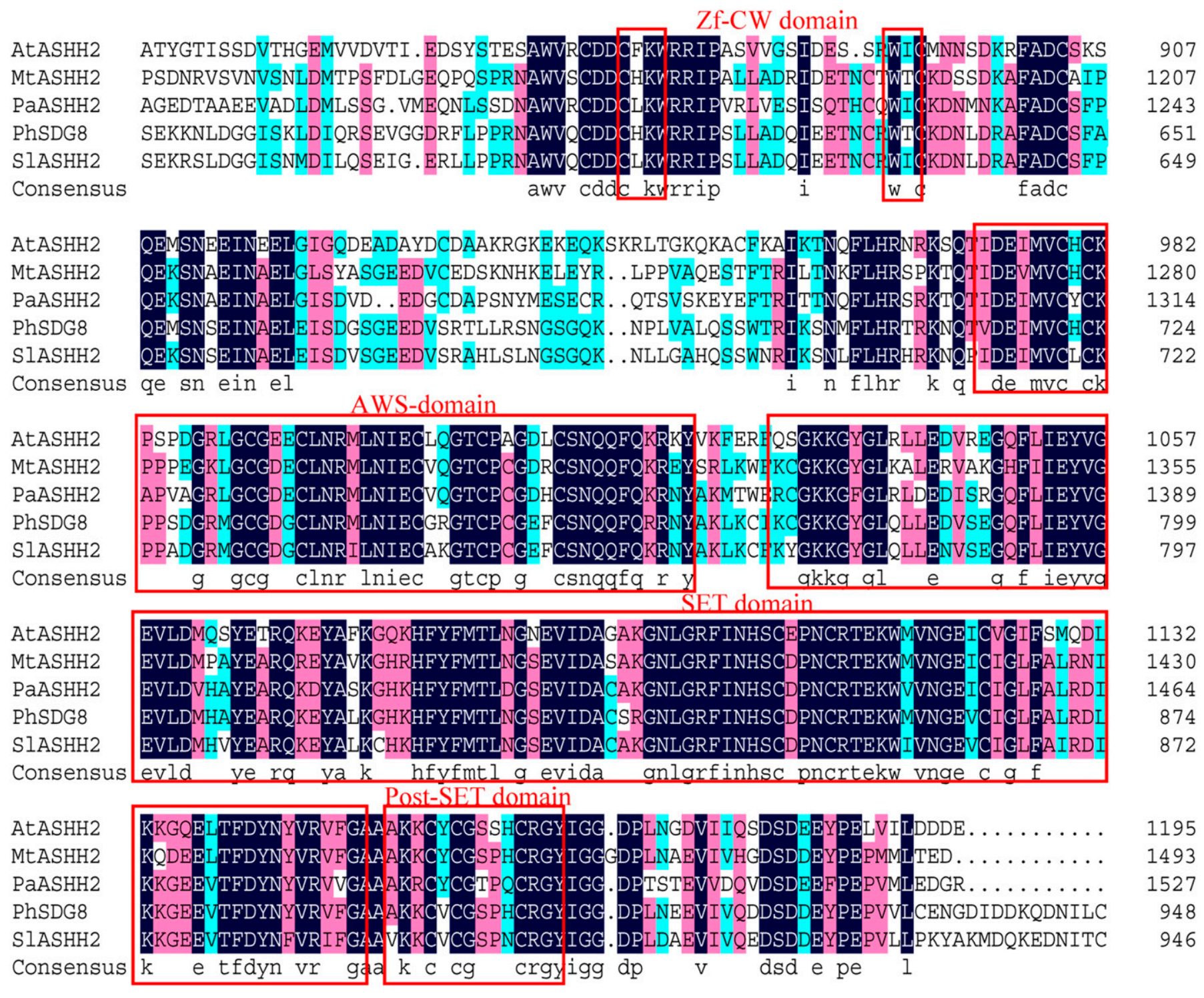

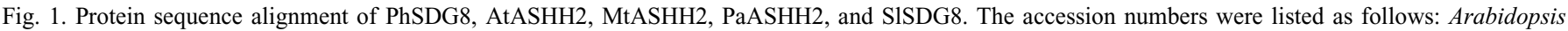
thaliana AtASHH2 (AT1G77300.1), Medicago truncatula MtASHH2 (XP_024634357.1), Populus alba PaASHH2 (TKS00672.1), Solanum lycopersicum SISDG8 (XP_010322942). Four functional domains, zf-CW, AWS, SET, and Post SET, are marked by red frames.

the stop codon, was amplified using the primers SDG8-F-SL/ SDG8-R-SL (Table 1), and then cloned into pCAMBIA1300 vector using ClonExpress ${ }^{\circledR}$ II One Step Cloning Kit (Vazyme, Nanjing, China), producing the PhSDG8-GFP construct under the control of the 35S promoter. The Agrobacterium tumefaciens containing 35S::PhSDG8-GFP was injected into the abaxial side of Nicotiana benthamiana leaves ( 2 weeks old). After culturing at $25^{\circ} \mathrm{C}$ for $48 \mathrm{~h}$, the fluorescence was observed by confocal laser scanning microscopy (FV1200; Olympus, Tokyo, Japan).

ARABIDOPSIS TRANSFORMATION AND PHENOTYPE ANALYSIS. The full-length of $P h S D G 8$ sequence was amplified with SDG8-FSL/PhSDG8-R-OE using KOD-Plus-Neo DNA Polymerase (Toyobo) (Table 1). The obtained sequence was ligated to pCAMBIA1300 vector using ClonExpress ${ }^{\circledR} I I$ One Step Cloning Kit (Vazyme), producing the 35S::PhSDG8 construct. The recombinant plasmid was transformed into $A$. tumefaciens by heat shock. Finally, the construct was introduced into homo- zygous sdg8-2 mutants through the A. tumefaciens-mediated flower-dipping method (Clough and Bent, 1998). Seeds were collected from the infiltrated plants and selected on a Murashige and Skoog (MS) medium supplemented with $50 \mathrm{mg} \cdot \mathrm{L}^{-1}$ kanamycin. Kanamycin-resistant plants were transferred to soil $15 \mathrm{~d}$ after germination and were grown in a growth chamber. The number of rosette branches (bud length $\geq 10$ $\mathrm{mm})$ and the height of the main stems were scored for phenotype analysis.

CONSTRUCTION AND TRANSFORMATION OF VIRUS-INDUCED GENE SILENCING (VIGS) VECTOR. Tobacco rattle virus (TRV)-based vectors, pTRV1 (pYL192) and pTRV2 (pYL156) were used in this study (Cao et al., 2015). The PhSDG8 (300 bp) fragment was amplified with primers SDG8-vigs-F/SDG8vigs-R. Then the PhSDG8 fragment was digested using BamHI and EcoRI restriction enzymes and cloned into pTRV2 to generate pTRV2-PhSDG8. The recombinant plasmid-carrying target gene fragment and empty vector as 


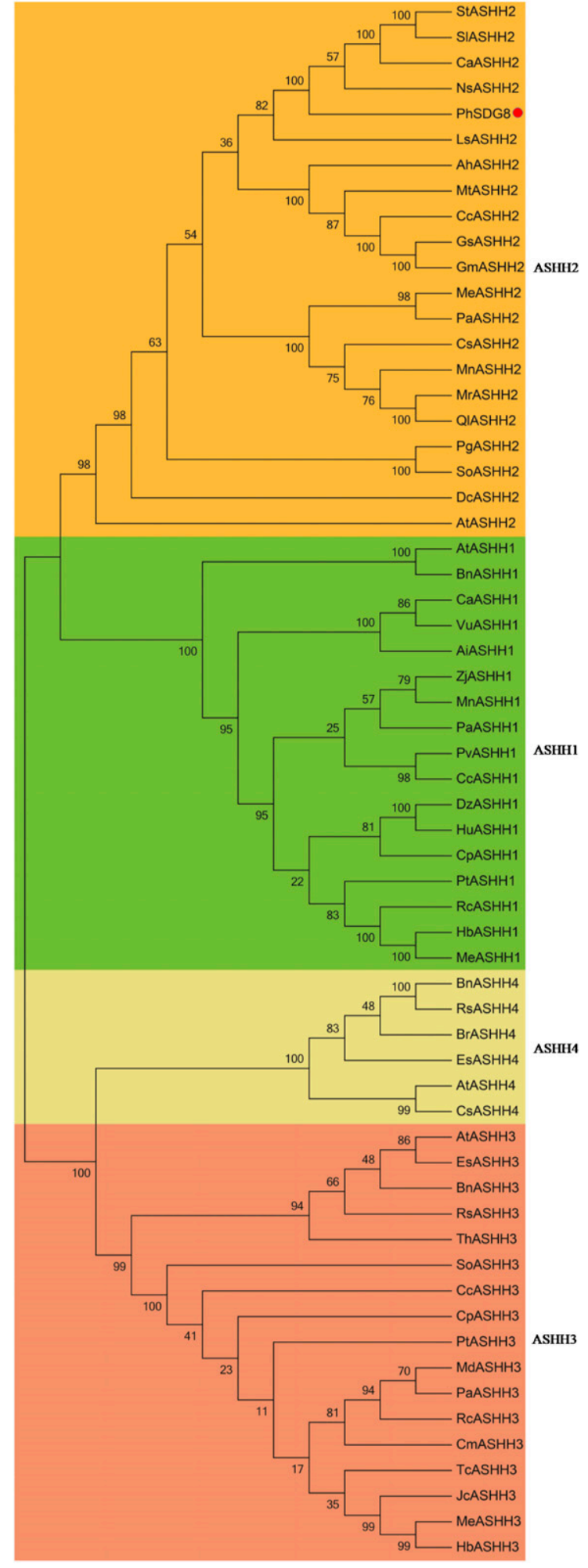

Fig. 2. Phylogenetic analysis of ASH1s. The phylogenetic tree was constructed using the neighbor-joining method. Numbers above branches indicate bootstrap support percentages from 1000 replicates. The accession numbers of selected ASH1s were listed as follows: Morella rubra MrASHH2 (KAB1203139.1), Quercus lobata Q1ASHH2 (XP_030933677.1), Citrus sinensis CsASHH2 (XP_024952142.1), Manihot esculenta MeASHH2 (XP_021598387.1), Morus notabilis MnASHH2 (XP_024019152.1), Lactuca sativa LsASHH2 (XP_023731848.1), Nicotiana sylvestris NsASHH2 (XP_009803898.1), Capsicum annuum CaASHH2 (XP_016575229.1), Solanum tuberosum StASHH2 (XP_006365937.1), Arachis hypogaea AhASHH2 control (pTRV2-0) were transformed into the A. tumefaciens strain GV3101 by a freeze-thaw method. According to Broderick's method, a single colony for each construct was selected for shaking culture to an optical density at $600 \mathrm{~nm}$ $\left(\mathrm{OD}_{600}\right)$ of $0.8-1.0$. Cells were collected and resuspended in media (10 mm 4-morpHolineethanesulfonic acid, $10 \mathrm{~mm}$ $\mathrm{MgCl}_{2}$, and $200 \mu \mathrm{M}$ acetosyringone) to an $\mathrm{OD}_{600}$ of $1.8-$ 2.0. Then, pTRV1 cultures were mixed with pTRV2PhSDG8 (or TRV2-0) cultures in a 1:1 ratio (Broderick and Jones, 2014). For leaf agroinfiltration, agrobacterium cultures were introduced into leaves of petunia seedlings selected randomly at the four-to-six leaf stage using syringes. After infiltration, seedlings were grown in a culturing room. Sixty days later, the number of branches (bud length $\geq 10 \mathrm{~mm}$ ) was counted; also the height of the plants was measured.

Statistical analysis. All the experiments in this study were repeated three times. Data were presented as mean value \pm SD. Tukey's tests were performed for statistical analyses of phenotypic and expression data.

\section{Results}

Cloning of PhSDG8 and protein structure analysis. Primers were designed based on the published genome sequences of Petunia inflata to obtain the full length of PhSDG8 sequence. The gene encoded 1689 amino acids with a relative molecular weight of $185.89 \mathrm{kDa}$ and an isoelectric point of 6.92, while the relative molecular weight of Arabidopsis SDG8 was $191.22 \mathrm{kDa}$ and the isoelectric point was 5.31 . The instability index of PhSDG8 was computed to be 55.41. The relatively high concentration of amino acids in the protein was serine $(11.6 \%)$, leucine $(8.2 \%)$, glycine $(7.7 \%)$, and lysine $(6.9 \%)$. The total number of negatively charged residues (aspartic acid + glutamic acid) and positively charged resi-

Fig. 2. (Continued). (XP_025666034.1), Cajanus cajan CcASHH2 (XP 020212539.1), Glycine soja GsASHH2 (RZC18189.1), Glycine max GmASHH2 (XP_014631840.1), Punica granatum PgASHH2 (XP_031394924.1), Syzygium oleosum SoASHH2 (XP_030476498.1), Dendrobium catenatum DcASHH2 (PKU81992.1), Arabidopsis thaliana AtASHH1 (AT1G76710.1), Brassica napus BnASHH1 (XP_013717811.1), Cicer arietinum CaASHH1 (XP_004496445.1), Vigna unguiculata VuASHH1 (XP_027937757.1), Arachis ipaensis AiASHH1 (XP_016176356.1), Ziziphus jujuba ZjASHH1 (XP_015886513.1), MnÁSHH1 (XP_024023788.1), Prunus avium PaASHH1 (XP_021817357.1), Pistacia vera PvASHH1 (XP_031250764.1), Citrus clementina CcASHH1 (XP_024040947.1), Durio zibethinus DzASHH1 (XP 022773422.1), Herrania umbratica HuASHH1 (XP_021284799.1), Carica papaya CpASHH1 (XP_021898665.1), Populus trichocarpa PtASHH1 (XP 002306713.1), Ricinus communis RcASHH1 (XP_002528669.1), Hevea brasiliensis HbASHH1 (XP_021650631.1), MeĀSHH1 (XP_021630030.1), BnASHH4 (XP_013659981.1), Raphanus sativus RsASHH4 (XP_018488040.1), Brassica rapa BrASHH4 (XP_009104235.1), Eutrema salsugineum EsASHH4 (XP_024012774.1), AtASHH4 (NP 001326226.1$)$, Camelina sativa CsASHH4 (XP_019082925.1), AtASHH3 (NP_566010.1), EsASHH3 (XP_006397594.1), BnASHH3 (XP_013687016.1), RsASHH3 (XP_018461825.1), Tarenaya hassleriana ThASHH3 (XP_010527044.1), SoASHH3 (XP 030456216.1), CcASHH3 (XP 024045341.1), CpASHH3 (XP_021907030.1), PtASHH3 (XP_024444632.1), Malus domestica MdASHH3 (XP 008394239.1), PaASHH3 (XP 021814053.1), Rosa chinensis RcASHH3 (XP_024185735.1), Cucurbita maxima CmASHH3 (XP_022969811.1), Theobroma cacao TcASHH3 (XP_007022844.1), Jatropha curcas JcASHH3 (XP_012081879.1), MeASHH3 (XP_021625182.1), and HbASHH3 (XP_021664231.1). PhSDG8 is marked with a red dot. 
dues (arginine + lysine) were 222 and 218, respectively. Aliphatic index was 68.85, and the grand average of hydropathicity (GRAVY) was -0.663 . The amino acid sequence of PhSDG8 was analyzed by SMART software (Letunic et al., 2012). The results showed that PhSDG8 contained Zf-CW (aa 614-617 and 635-637), AWS (aa 715-767), SET (aa 773-892), and Post SET domain (aa 894-907) (Fig. 1), which are all the characteristic of SDG8 (He et al., 2010; Liu and Huang, 2018). The amino acid sequence of PhSDG8 shared $71 \%$ identity with those of S1SDG8 (Fig. 1). Alignment of the amino acid sequence encoded by PhSDG8 with those of other SDG8 proteins from Arabidopsis, Medicago truncatula, Populus alba, and Solanum lycopersicum showed that sequences were conserved mostly in the four domains (Fig. 1).

Phylogenetic analysis of PhSDG8. To determine the genetic relationship between PhSDG8 and other SDG8s, phylogenetic analyses were performed (Fig. 2). A total of 60 ASH1 SET protein sequences were collected from 43 species. The phylogenetic tree displayed four major clades that corresponded to the ASHH1, ASHH2, ASHH3, and ASHH4 subfamilies, while the PhSDG8 protein was clustered in ASHH2 clade, with the same clade with Solanaceae plants. PhSDG8 was most closely related to NsASHH2.

Tissue-SPECIFIC EXPRESSION ANALYSIS OF PHSDG8. To analyze the expression of $P h S D G 8$ in different tissues, the relative expression level of $P h S D G 8$ in roots, stems, leaves, axils, and apices of petunia were detected (Fig. 3). The results showed that PhSDG8 was expressed in all detected tissues, with the highest expression in stems and the lowest in axil. The expression level of PhSDG8 in apex, axil, roots, and stems were $0.42,0.21,0.94$, and 1.8 of leaves, respectively.

Subcellular localization of PhSDG8. To clarify the function of the PhSDG8, we analyzed the subcellular localization of PhSDG8. A pCAMBIA1300 vector containing PhSDG8 fused to Green Fluorescent Protein (GFP) at its N terminus was constructed in this research. We used pCAMBIA1300 as a control. After transient transformation into $N$. benthamiana, GFP drived by $35 \mathrm{~S}$ had green fluorescence on the cell membrane and nucleus, while PhSDG8 fused with GFP only produced green fluorescence in the nucleus, indicating that PhSDG8 was localized in the nucleus (Fig. 4).

Functional analysis of PhSDG8. To analyze the function of PhSDG8, 35S::PhSDG8 vector was constructed and transformed into Arabidopsis sdg8-2. The obtained transgenic lines were used for branch phenotypic analysis, and the leaves of transgenic lines were used to detect expression level of $P h S D G 8$. It was found that $P h S D G 8$ was detected in five transgenic lines, but not in the WT and $s d g 8-2$ mutants (Fig. 5D). Overexpression of $P h S D G 8$ restored the branch and height phenotype of $s d g 8-2$ to the wild type (Fig. $5 \mathrm{~A}-\mathrm{C}$ ), suggesting that $P h S D G 8$ can regulate branch development of Arabidopsis.

Silencing of PhSDG8 in petunia. To confirm PhSDG8 suppression at the molecular level, the expression level of $P h S D G 8$ was determined by qRT-PCR. The average expression level of $P h S D G 8$ in pTRV2-0 plants was 4-fold higher than that in pTRV2-PhSDG8 seedlings (Fig. 6B), which indicated that $P h S D G 8$ was silenced. Silencing of $P h S D G 8$ led to an increase in the number of branches of petunia from 1.4 to 4.4 (Fig. 6C), and a decrease in plant height from 596 to $413 \mathrm{~mm}$ (Fig. 6D), indicating that the expression level of PhSDG8 was correlated with the number of branches of petunia. The expression of PhBRC1, PhRAX1, PhRAX2, PhRAX3, PhLAS, PhROX,

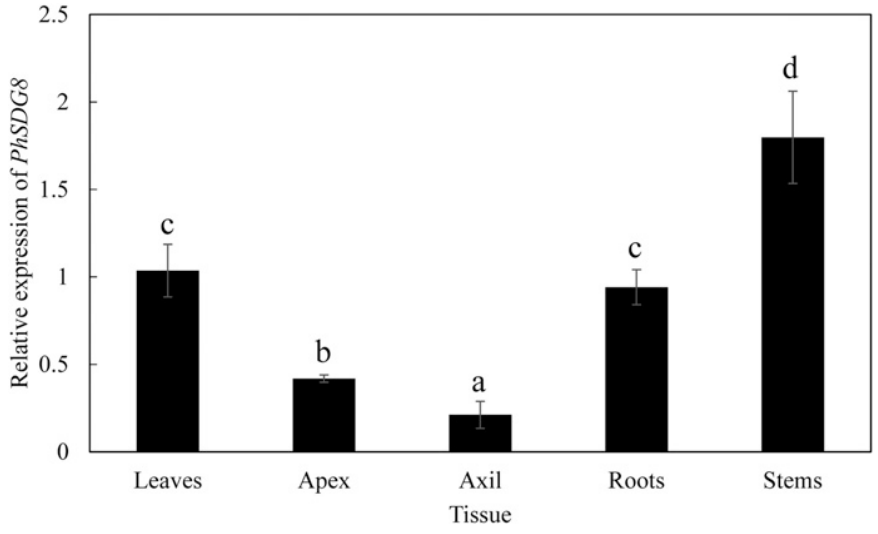

Fig. 3. Expression analysis of $P h S D G 8$ in different tissues of petunia. All samples were taken from three pools of 12 plantlets. Detection of $\beta$-actin was used as a control. Error bars show the standard deviations (SD) of three biological replicates. Lowercase letters a and $\mathrm{b}$ indicate significant differences via Tukey's test at $P<0.05$.

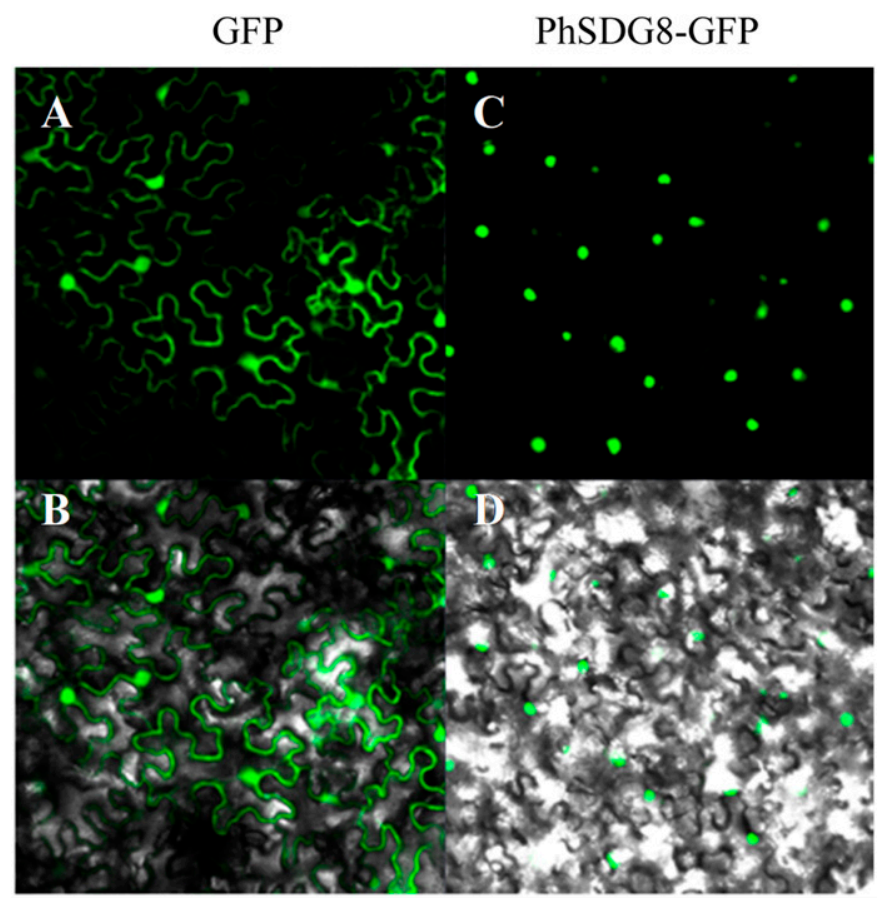

Fig. 4. Subcellular localization of PhSDG8-GFP protein in tobacco epidermal cells. (A, C) Fluorescence images of PhSDG8-GFP fusion protein and control are shown. (B, D) The merged images of PhSDG8-GFP fusion protein and control are shown.

PhWRKY71, and PhUGT74E2 were detected in three lines that infiltrated with VIGS vectors carrying PhSDG8 inside (Fig. 6E). The results showed that only the PhUGT74E2 expression level was significantly up-regulated, which suggested that $P h S D G 8$ could affect the development of branches by regulating the expression of PhUGT74E2.

\section{Discussion}

Proteins containing the conserved SET domain can be found in various species. SET domain proteins are categorized into seven classes: Enhancer of zeste [E (z)], ASH1, Trithorax (Trx), Suppressor variegation [Su (var)], and so on (Chen et al., 2020). 

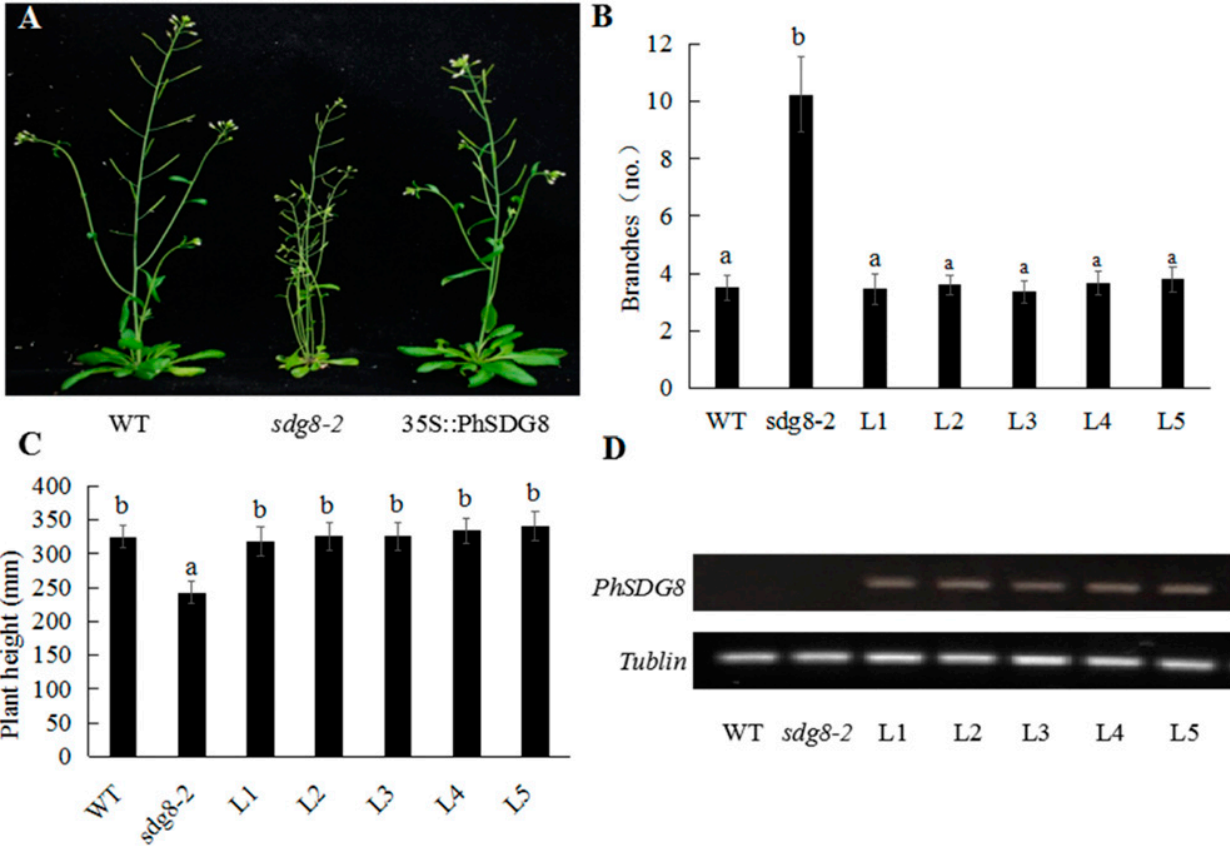

Fig. 5. Overexpression of PhSDG8 restored the phenotype of Arabidopsis thaliana sdg8-2. (A) Comparison of phenotypes of the $A$. thaliana wild type (WT), sdg8-2, and $s d g 8-2$ transformed with 35 S::PhSDG8. (B) The number of secondary rosette branches produced by WT, sdg8-2, and five independent homozygous lines carrying 35S::PhSDG8 are shown. L represents transgenic line, and $n=20$. (C) The height of the main branches of WT, $s d g 8-2$, and five independent transgenic lines are shown. (D) Transcripts of PhSDG8 were assayed by reverse transcriptase polymerase chain reaction (RT-PCR) from total RNA of leaves. Detection of the Tublin transcript was used as a control. Lowercase letters a and b indicate significant differences via Tukey's test at $P<$ 0.05 .

In Arabidopsis, there are five ASH1 SET domain proteins: SDG26/ASHH1, SDG8/ASHH2, SDG7/ASHH3, SDG24/ ASHH4, and SDG4/ASHR3 (Zhu et al., 2013). Several recent studies found that $S D G 8$ was involved multiple biological processes, such as shoot branching (Dong et al., 2008), carotenoid composition (Cazzonelli et al., 2009), ovule and anther development (Grini et al., 2009), response to a mechanical stimulus (Cazzonelli et al., 2014), and embryogenesis (Cheng et al., 2018). Therefore, $S D G 8$ is an important gene for regulating plant growth and development. However, although there is some research on SDG8 in Arabidopsis, no reports are found in petunia.

In this study, an ortholog of SDG8 was cloned from petunia cultivar Mitchell Diploid. Sequence analysis revealed that, in addition to the presence of canonical domains (AWS, SET, post-SET), PhSDG8 contained a Zf-CW domain, which was unique to $S D G 8$ among all SET proteins. The conserved Cys-X$\mathrm{X}$-Trp and Trp-X-Cys sequence motifs in Zf-CW domain were considered to bind to DNA sequences or protein domains, and specifically target histone methylation (Christopher et al., 2009). In addition, studies have shown that the $\mathrm{Zf}-\mathrm{CW}$ domain of SDG8 preferentially binds monomethylated H3K4 (Liu and Huang, 2018). A phylogenetic tree and sequence alignment showed that PhSDG8 had the same origin as $\mathrm{ASHH} 2$ protein of other species, and subsequently diverged at different phases of later evolution, indicating PhSDG8 indeed belonged to the ASHH2 class.

Expression analysis showed that $P h S D G 8$ had higher expression in stems than in other detected tissues. This was consistent with the conclusion that $B n S D G 8 A$ and $B n S D G 8 B$ were highly expressed in Brassica napus stems, compared with the expression in roots and leaves (Jiang et al., 2018). Study in Arabidopsis showed that the expression of $S D G 8$ in the shoot apical meristem was higher than that in leaves (Cazzonelli et al., 2010). Therefore, it appears that the expression patterns of PhSDG8 are similar, and different compared with other species. Subcellular localization analysis showed that PhSDG8 was localized in the nucleus, indicating that PhSDG8 was a protein that acted in the nucleus, which is consistent with the conclusion of SDG8 in Arabidopsis (Xu et al., 2008). Similar to BnSDG8 gene (Jiang et al., 2018), overexpression of PhSDG8 in Arabidopsis completely restored the phenotype of $s d g 8-2$, including branch number and plant height. This result not only demonstrates that $P h S D G 8$ is a functional gene for regulating shoot branching but also shows the function conservation of $S D G 8 s$ among different species.

TRV has been successfully used as a VIGS vector in many species, although the experimental methods vary among species (Broderick and Jones, 2014). In this study, $P h S D G 8$ was effectively silenced in petunia. The decrease of $P h S D G 8$ expression level led to the increase of the number of branches in petunia, accompanied by the decrease of plant height and early flowering. These results suggest $P h S D G 8$ was an inhibitor of branch development and flowering regulation. $B R C 1, R A X 1, R A X 2, R A X 3$, $P h L A S, R O X, W R K Y 71$, and UGT74E2 are important genes for regulating branching (Guo et al., 2015; Tognetti et al., 2010; Wang et al., 2018, 2019). Silencing of PhSDG8 only caused significant up-regulation of PhUGT74E2; while this had no effect on other genes, especially without causing any changes in PhBRC1 expression level, whose homologous genes have been confirmed to be involved in the response to multiple signals of regulating shoot branching (Barbier et al., 2019). The result indicated that $P h S D G 8$ acted independently of the above pathways. UGT74E2 is a member of the Arabidopsis glycosylation transferase family 1 (Family-1) and encodes 453 amino acids, which can catalyze auxin for glycosylation (Rehman et al., 2018). Studies have shown that the overexpression of $U G T 74 E 2$ causes a significant increase in the number of branches (Tognetti et al., 2010). Therefore, it is speculated that $P h S D G 8$ can disrupt the dynamic balance of auxin by regulating the transcription of $P h U G T 74 E 2$ and cause abnormal development of plant branches, while the regulation mechanism of $P h S D G 8$ to $P h U G T 74 E 2$ still needs further study.

Our results presented here reveal the existence of PhSDG8 and function of regulating shoot branching in petunia. It provides not only the experimental basis for revealing the branching mechanism of petunia, but also offers the gene 
$\mathbf{A}$

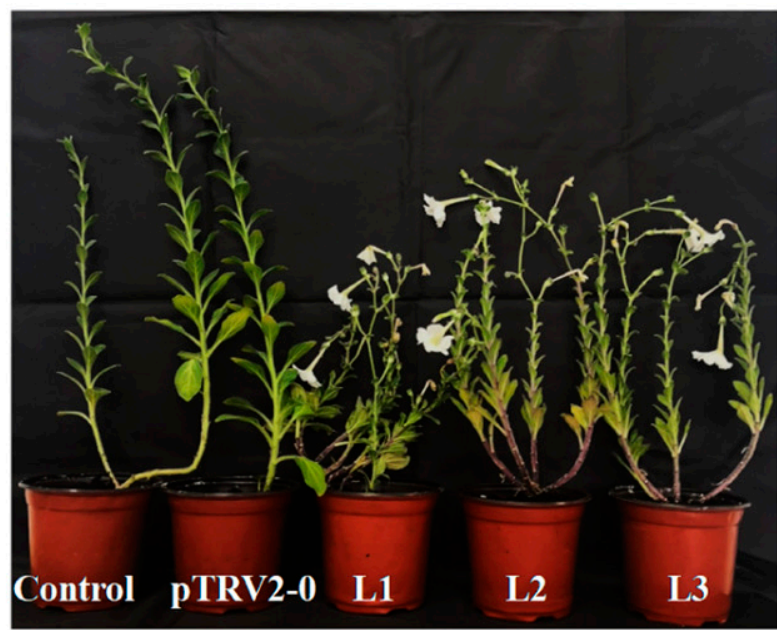

C

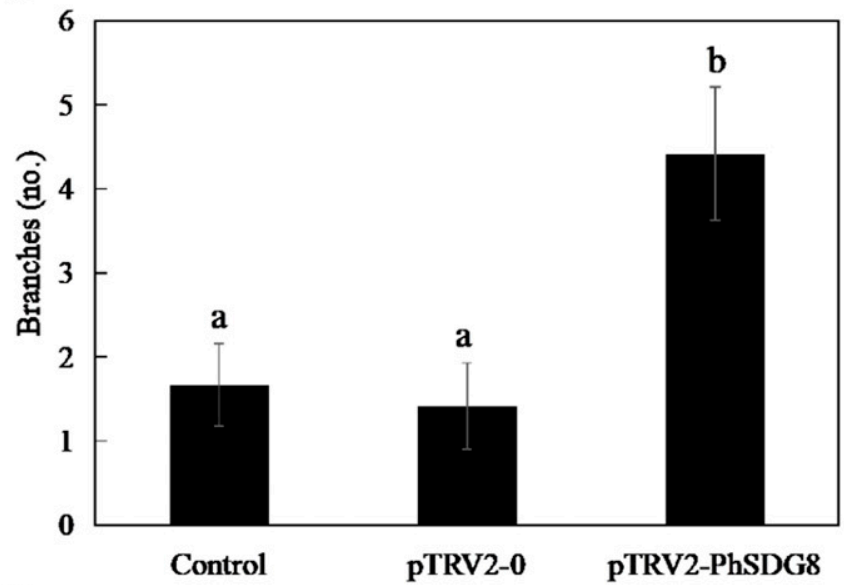

B

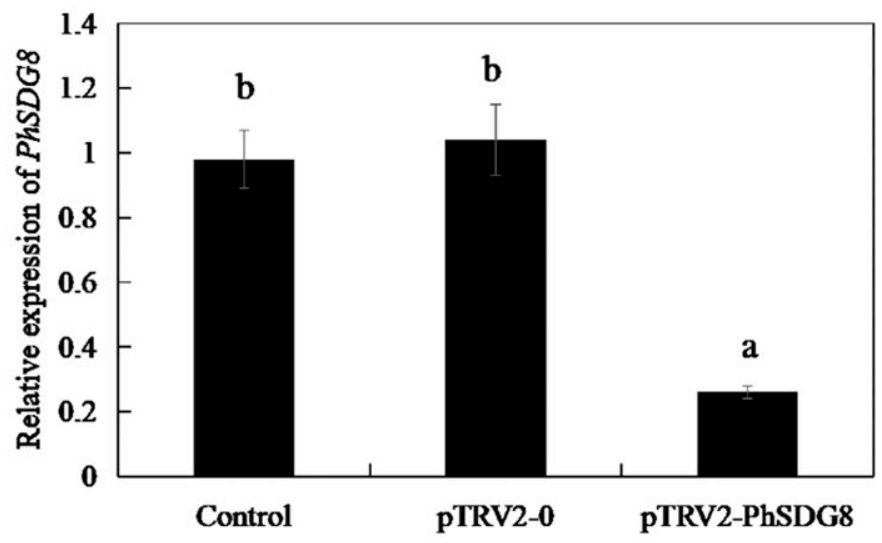

D

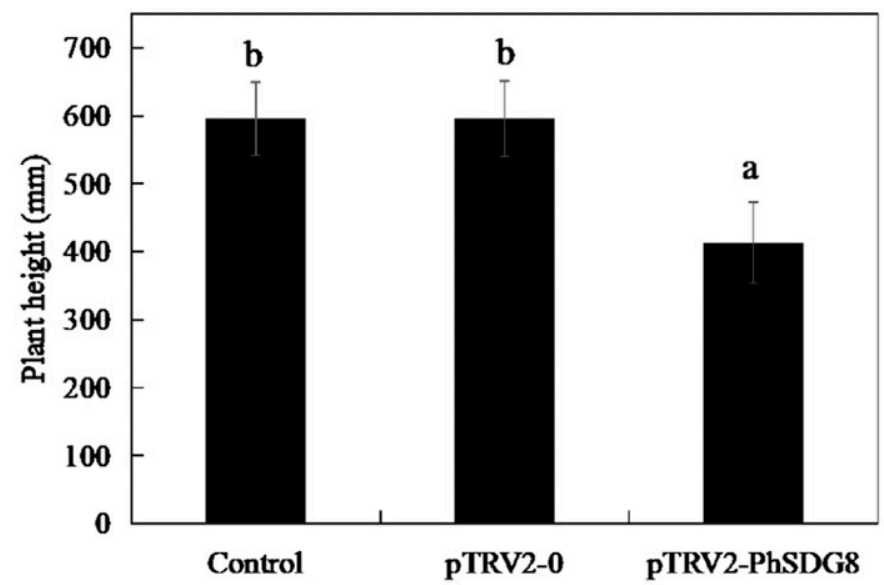

$\mathbf{E}$

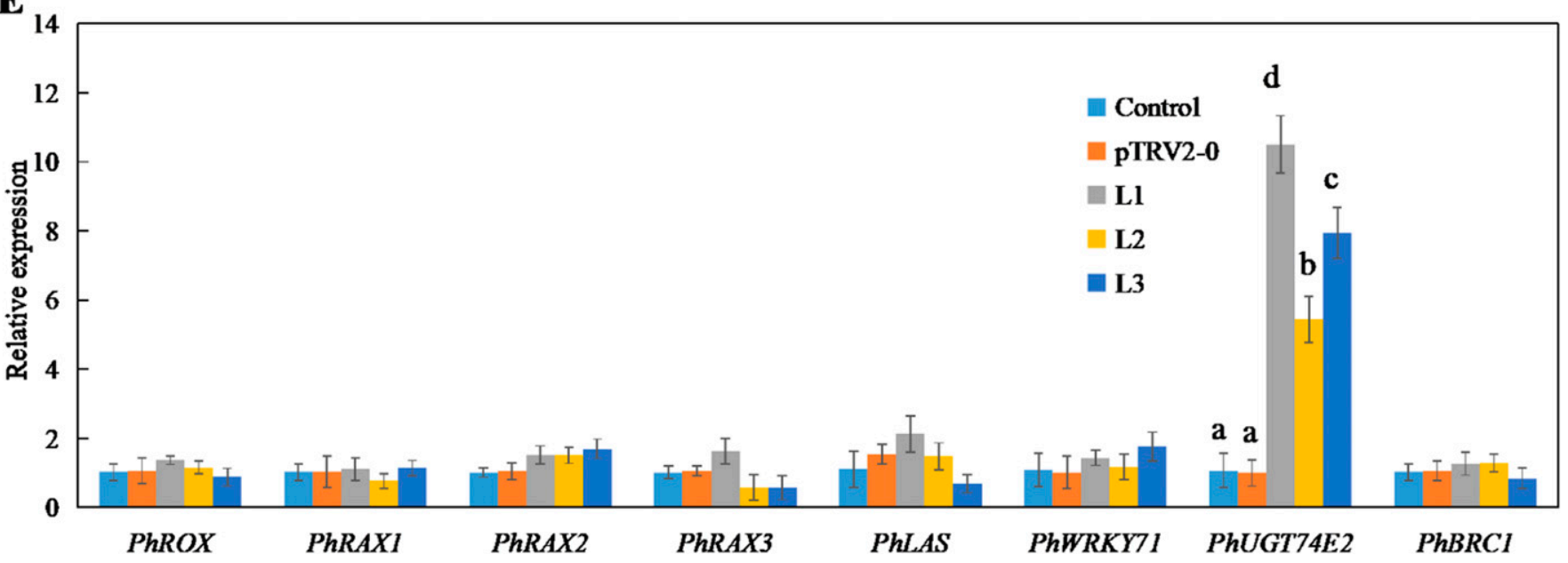

Fig. 6. Branch number phenotype and gene expression analysis of silencing of PhSDG8 in petunia. (A) Comparison of phenotypes of control and plants infiltrated with pTRV2-0 or pTRV2-PhSDG8. L1, 2, 3 represents different lines infiltrated with pTRV2-PhSDG8. (B) Transcripts of PhSDG8 were assayed by qRT-PCR from total RNA of leaves. Detection of the $\beta$-actin transcript was used as a control. Error bars show the SD of three biological replicates. Control, pTRV2-0, and pTRV2-PhSDG8 were all taken from three pools of 12 plantlets. (C) The branch numbers of control and plants infiltrated with pTRV2-0 or pTRV2-PhSDG8 are shown $(\mathrm{n}=12)$. (D) The height of the main branches of control and plants infiltrated with pTRV2-0 or pTRV2-PhSDG8 are shown (n=12). (E) Expression levels of PhROX, PhRAX1, PhRAX2, PhRAX3, PhLAS, PhWRKY71, PhUGT74E2, and PhBRC1 in control and plants infiltrated with pTRV2-0 or pTRV2-PhSDG8 were detected. Detection of the $\beta$-actin transcript was used as a control. Error bars of control and pTRV2-0 show the sD of three biological replicates, while error bars of L1, L2, and L3 show the SD of three technical replicates. Lowercase letters indicate significant differences via Tukey's test $(P<0.05)$, while groups without letters indicate no significant difference. 
resource for the use of $P h S D G 8$ to improve the plant architecture.

\section{Literature Cited}

Barbier, F.F., E.A. Dun, S.C. Kerr, T.G. Chabikwa, and C.A. Beveridge. 2019. An update on the signals controlling shoot branching. Trends Plant Sci. 24:220-236.

Bombarely, A., M. Moser, A. Amrad, M. Bao, L. Bapaume, C.S. Barry, M. Bliek, M.R. Boersma, L. Borghi, R. Bruggmann, M. Bucher, N. D'Agostino, K. Davies, U. Druege, N. Dudareva, M. Egea-Cortines, M. Delledonne, N. Fernandez-Pozo, P. Franken, L. Grandont, J.S. Heslop-Harrison, J. Hintzsche, M. Johns, R. Koes, X. Lv, E. Lyons, D. Malla, E. Martinoia, N.S. Mattson, P. Morel, L.A. Mueller, J. Muhlemann, E. Nouri, V. Passeri, M. Pezzotti, Q. Qi, D. Reinhardt, M. Rich, K.R. Richert-Pöggeler, T.P. Robbins, M.C. Schatz, M.E. Schranz, R.C. Schuurink, T. Schwarzacher, K. Spelt, H. Tang, S.L. Urbanus, M. Vandenbussche, K. Vijverberg, G.H. Villarino, R.M. Warner, J. Weiss, Z. Yue, J. Zethof, F. Quattrocchio, T.L. Sims, and C. Kuhlemeier. 2016. Insight into the evolution of the Solanaceae from the parental genomes of Petunia hybrida. Nat. Plants 2:16074, doi: 10.1038/nplants.2016.74.

Broderick, S.R. and M.L. Jones. 2014. An optimized protocol to increase virus-induced gene silencing efficiency and minimize viral symptoms in Petunia. Plant Mol. Biol. Rpt. 32:219-233.

Cao, K., L. Cui, X. Zhou, L. Ye, Z. Zou, and S. Deng. 2015. Four tomato FLOWERING LOCUS T-Like proteins act antagonistically to regulate floral initiation. Front. Plant Sci. 6:1213, doi: 10.3389/ fpls.2015.01213.

Cazzonelli, C.I., A.J. Cuttriss, S.B. Cossetto, W. Pye, P. Crisp, J. Whelan, E.J. Finnegan, C. Turnbull, and B.J. Pogson. 2009. Regulation of carotenoid composition and shoot branching in Arabidopsis by a chromatin modifying histone methyltransferase, SDG8. Plant Cell 21:39-53.

Cazzonelli, C.I., N. Nisar, A.C. Roberts, K.D. Murray, J.O. Borevitz, and B.J. Pogson. 2014. A chromatin modifying enzyme, SDG8, is involved in morphological, gene expression, and epigenetic responses to mechanical stimulation. Front. Plant Sci. 5:533, doi: 10.3389/fpls.2014.00533.

Cazzonelli, C.I., A.C. Roberts, M.E. Carmody, and B.J. Pogson. 2010. Transcriptional control of SET DOMAIN GROUP 8 and CAROTENOID ISOMERASE during Arabidopsis development. Mol. Plant 3:174-191.

Chen, D.H., H.L. Qiu, Y. Huang, L. Zhang, and J.P. Si. 2020. Genomewide identification and expression profiling of SET DOMAIN GROUP family in Dendrobium catenatum. BMC Plant Biol. 20:40, doi: 10.1186/s12870-020-2244-6.

Cheng, J., Y. Zhang, Z. Li, T. Wang, X. Zhang, and B. Zheng. 2018. A lariat-derived circular RNA is required for plant development in Arabidopsis. Sci. China Life Sci. 61:204-213.

Christopher, C.I., T. Millar, E.J. Finnegan, and B.J. Pogson. 2009. Promoting gene expression in plants by permissive histone lysine methylation. Plant Signal. Behav. 4:484-488.

Clough, S.J. and A.F. Bent. 1998. Floral-dip: A simplified method for Agrobacterium-mediated transformation of Arabidopsis thaliana. Plant J. 16:735-743.

Dal Santo, S., M. Fasoli, E. Cavallini, G.B. Tornielli, M. Pezzotti, and S. Zenoni. 2011. PhEXPA1, a Petunia hybrida expansin, is involved in cell wall metabolism and in plant architecture specification. Plant Signal. Behav. 6:2031-2034.

Dong, L., A. Ishak, J. Yu, R. Zhao, and L. Zhao. 2013. Identification and functional analysis of three $M A X 2$ orthologs in chrysanthemum. J. Integr. Plant Biol. 55:434-442.

Dong, G., D.P. Ma, and J. Li. 2008. The histone methyltransferase SDG8 regulates shoot branching in Arabidopsis. Biochem. Biophys. Res. Commun. 373:659-664.

Drummond, R.S.M., B.J. Janssen, Z. Luo, C. Oplaat, S.E. Ledger, M.W. Wohlers, and K.C. Snowden. 2015. Environmental control of branching in petunia. Plant Physiol. 168:735-751.
Drummond, R.S.M., N.M. Martínez-Sánchez, B.J. Janssen, K.R. Templeton, J.L. Simons, B.D. Quinn, S. Karunairetnam, and K.C. Snowden. 2009. Petunia hybrida CAROTENOID CLEAVAGE DIOXYGENASE 7 is involved in the production of negative and positive branching signals in petunia. Plant Physiol. 151:1867-1877. Drummond, R.S.M., H. Sheehan, J.L. Simons, N.M. MartínezSánchez, R.M. Turner, J. Putterill, and K.C. Snowden. 2011. The expression of petunia strigolactone pathway genes is altered as part of the endogenous developmental program. Front. Plant Sci. 2:115, doi: $10.3389 /$ fpls.2011.00115.

Dun, E.A., A.D.S. Germain, C. Rameau, and C.A. Beveridge. 2012. Antagonistic action of strigolactone and cytokinin in bud outgrowth control. Plant Physiol. 158:487-498.

Fambrini, M., M. Salvini, and C. Pugliesi. 2017. Molecular cloning, phylogenetic analysis, and expression patterns of LATERAL SUPPRESSOR-LIKE and REGULATOR OF AXILLARY MERISTEM FORMATION-LIKE genes-in sunflower (Helianthus annuus L.). Dev. Genes Evol. 227:159-170.

Gasteiger, E., C. Hoogland, A. Gattiker, M.R. Wilkins, R.D. Appel, and A. Bairoch. 2005. Protein identification and analysis tools on the ExPASy server, p. 571-607. In: J.M. Walker (ed.). The proteomics protocols handbook. Humana Press, Totowa, NJ.

Grini, P.E., T. Thorstensen, V. Alm, G. Vizcay-Barrena, S.S. Windju, T.S. Jørstad, Z.A. Wilson, and R.B. Aalen. 2009. The ASH1 HOMOLOG 2 (ASHH2) histone $\mathrm{H} 3$ methyltransferase is required for ovule and anther development in Arabidopsis. PLoS One 4:e7817, doi: 10.1371/journal.pone.0007817.

Guo, D., J. Zhang, X. Wang, X. Han, B. Wei, J. Wang, B. Li, H. Yu, Q. Huang, H. Gu, L.J. Qu, and G. Qin. 2015. The WRKY transcription factor WRKY71/EXB1 controls shoot branching by transcriptionally regulating $R A X$ genes in arabidopsis. Plant Cell 27:3112-3127.

Hamiaux, C., R.S.M. Drummond, B.J. Janssen, S.E. Ledger, J.M. Cooney, R.D. Newcomb, and K.C. Snowden. 2012. DAD2 is an $\alpha / \beta$ hydrolase likely to be involved in the perception of the plant branching hormone, strigolactone. Curr. Biol. 22:2032-2036.

He, F., T. Umehara, K. Saito, T. Harada, S. Watanabe, T. Yabuki, T. Kigawa, M. Takahashi, K. Kuwasako, K. Tsuda, T. Matsuda, M. Aoki, E. Seki, N. Kobayashi, P. Güntert, S. Yokoyama, and Y. Muto. 2010. Structural insight into the zinc finger $\mathrm{CW}$ domain as a histone modification reader. Structure 18:1127-1139.

Jiang, L., D. Li, L. Jin, Y. Ruan, W.H. Shen, and C. Liu. 2018. Histone lysine methyltransferases BnaSDG8. A and BnaSDG8. C are involved in the floral transition in Brassica napus. Plant J. 95:672-685.

Kim, S.Y., Y. He, Y. Jacob, Y.S. Noh, S. Michaels, and R. Amasino. 2005. Establishment of the vernalization-responsive, winter-annual habit in Arabidopsis requires a putative histone H3 methyltransferase. Plant Cell 17:3301-3310.

Kretzschmar, T., W. Kohlen, J. Sasse, L. Borghi, M. Schlegel, J.B. Bachelier, D. Reinhardt, R. Bours, H.J. Bouwmeester, and E. Martinoia. 2012. A petunia ABC protein controls strigolactonedependent symbiotic signalling and branching. Nature 483:341-344. Letunic, I., T. Doerks, and P. Bork. 2012. SMART 7: Recent updates to the protein domain annotation resource. Nucleic Acids Res. 40:302305 .

Liu, B., A. Berr, C. Chang, C. Liu, W.H. Shen, and Y. Ruan. 2016. Interplay of the histone methyltransferases SDG8 and SDG26 in the regulation of transcription and plant flowering and development. Biochimica et Biophysica Acta (BBA) Gene Regulatory Mechanisms 1859:581-590.

Liu, Y. and Y. Huang. 2018. Uncovering the mechanistic basis for specific recognition of monomethylated $\mathrm{H} 3 \mathrm{~K} 4$ by the $\mathrm{CW}$ domain of Arabidopsis histone methyltransferase SDG8. J. Biol. Chem. 293:6470-6481.

Müller, D., G. Schmitz, and K. Theres. 2006. Blind homologous R2R3 $M y b$ genes control the pattern of lateral meristem initiation in Arabidopsis. Plant Cell 18:586-597.

Rehman, H.M., M.A. Nawaz, Z.H. Shah, J. Ludwig-Müller, G. Chung, M.Q. Ahmad, S.H. Yang, and S.I. Lee. 2018. Comparative genomic 
and transcriptomic analyses of Family-1 UDP glycosyltransferase in three Brassica species and Arabidopsis indicates stress-responsive regulation. Sci. Rep. 8:1-18.

Revel, S.M., J.J. Bart, Z. Luo, C. Oplaat, E.L. Susan, W.W. Mark, and C.S. Kimberley. 2015. Environmental control of branching in petunia. Plant Physiol. 168:735-751.

Shen, J., Y. Zhang, D. Ge, Z. Wang, W. Song, R. Gu, G. Che, Z. Cheng, R. Liu, and X. Zhang. 2019. CsBRC1 inhibits axillary bud outgrowth by directly repressing the auxin efflux carrier CSPIN3 in cucumber. Proc. Natl. Acad. Sci. USA 116:17105-17114.

Shimizu-Sato, S., M. Tanaka, and H. Mori. 2009. Auxin-cytokinin interactions in the control of shoot branching. Plant Mol. Biol. 69:429-435.

Snowden, K.C., A.J. Simkin, B.J. Janssen, K.R. Templeton, H.M. Loucas, J.L. Simons, S. Karunairetnam, A.P. Gleave, D.G. Clark, and H.J. Klee. 2005. The Decreased apical dominance1/Petunia hybrida CAROTENOID CLEAVAGE DIOXYGENASE 8 gene affects branch production and plays a role in leaf senescence, root growth, and flower development. Plant Cell 17:746-759.

Sui, P., J. Shi, X. Gao, W. Shen, and A. Dong. 2013. H3K36 methylation is involved in promoting rice flowering. Mol. Plant 6:975-977.

Tamura, K., D. Peterson, N. Peterson, G. Stecher, M. Nei, and S. Kumar. 2011. MEGA5: Molecular evolutionary genetics analysis using maximum likelihood, evolutionary distance, and maximum parsimony methods. Mol. Biol. Evol. 28:2731-2739.

Tognetti, V.B., O. Van Aken, K. Morreel, K. Vandenbroucke, B. Van De Cotte, I. De Clercq, S. Chiwocha, R. Fenske, E. Prinsen, W. Boerjan, B. Genty, K.A. Stubbs, D. Inzé, and F.V. Breusegem. 2010.
Perturbation of indole-3-butyric acid homeostasis by the UDPglucosyltransferase UGT74E2 modulates Arabidopsis architecture and water stress tolerance. Plant Cell 22:2660-2679.

Wang, M., M.A. Le Moigne, J. Bertheloot, L. Crespel, M.D. PerezGarcia, L. Ogé, S. Demotes-Mainard, L. Hamama, J.M. Davière, and S. Sakr. 2019. BRANCHED1: A key hub of shoot branching. Front. Plant Sci. 10:76, doi: 10.3389/fpls.2019.00076.

Wang, B., M. Smith Steven, and J. Li. 2018. Genetic regulation of shoot architecture. Annu. Rev. Plant Biol. 69:437-468.

$\mathrm{Xu}, \mathrm{L}$., Z. Zhao, A. Dong, L. Soubigou-Taconnat, J.P. Renou, A. Steinmetz, and W.H. Shen. 2008. Di- and tri- but not monomethylation on histone $\mathrm{H} 3$ lysine 36 marks active transcription of genes involved in flowering time regulation and other processes in Arabidopsis thaliana. Mol. Cell. Biol. 28:1348-1360.

Yang, F., Q. Wang, G. Schmitz, D. Müller, and K. Theres. 2012. The bHLH protein ROX acts in concert with RAX1 and LAS to modulate axillary meristem formation in arabidopsis. Plant J. Cell. Mol. Biol. 71:61-70.

Zenoni, S., M. Fasoli, G.B. Tornielli, S.T. Dal Santo, A. Sanson, P. de Groot, S. Sordo, S. Citterio, F. Monti, and M. Pezzotti. 2011. Overexpression of PhEXPAl increases cell size, modifies cell wall polymer composition and affects the timing of axillary meristem development in Petunia hybrida. New Phytol. 191:662-677.

Zhao, Z., Y. Yu, D. Meyer, C. Wu, and W.H. Shen. 2005. Prevention of early flowering by expression of FLOWERING LOCUS C requires methylation of histone H3K36. Nat. Cell Biol. 7:1256-1260.

Zhu, X., B. Wang, X. Kan, C. Chen, and C. Yu. 2013. Evolution of plant Ash1 SET genes: Structural divergence and functional differentiation. Genes Genomics 35:463-473. 